\title{
A Fractional Supervision Game Model of Multiple Stakeholders and Numerical Simulation
}

\author{
Rongwu Lu, ${ }^{1}$ Xinhua Wang, ${ }^{2}$ and Dan $\mathrm{Li}^{2}$ \\ ${ }^{1}$ College of Mathematics and Systems Science, Shandong University of Science and Technology, Qingdao 266590, China \\ ${ }^{2}$ College of Economics and Management, Shandong University of Science and Technology, Qingdao 266590, China
}

Correspondence should be addressed to Xinhua Wang; wangxinhua201@163.com

Received 10 February 2017; Revised 3 April 2017; Accepted 3 April 2017; Published 13 April 2017

Academic Editor: Vladimir Turetsky

Copyright (C) 2017 Rongwu Lu et al. This is an open access article distributed under the Creative Commons Attribution License, which permits unrestricted use, distribution, and reproduction in any medium, provided the original work is properly cited.

Considering the popular use of a certain kind of supervision management problem in many fields, we firstly build an ordinary supervision game model of multiple stakeholders. Secondly, a fractional supervision game model is set up and solved based on the theory of fractional calculus and a predictor-corrector numerical approach. Thirdly, the methods of phase diagram and time series graph were applied to simulate and analyse the dynamic process of the fractional order game model. Results of numerical solutions are given to illustrate our conclusions and referred to the practice.

\section{Introduction}

There is a typical kind of supervision game model used to describe the harmony, balance, and conflicts among the participating groups from different levels, such as coal mining workers, inspection team, and the government safety supervision department in colliery safety management system, or pollution companies, local environmental protection department and the supervisory department of environmental protection in environmental supervision system, and so forth. The characteristic of this kind of regulatory and supervision issues is that both the inspection and supervision behaviors are done to the same object (the producers). That is, not only does the internal quality inspection department of the enterprise check the standardization of the production process, but also, due to the social externality of the product, the superior administrative department or the community residents supervise the production process. In this way, workers, quality inspection departments and government departments, and other stakeholders will take a long-term dynamic game process. Many researchers have done a lot of study on this kind of problems [1-5] and have obtained plenty of theoretical achievements.

Actually, the supervision game is an extremely complex nonlinear system involving many factors. There are too many characteristics that cannot be described precisely by integral order calculus. Attributed to the fast development of computer technology and numerical simulation, the fractional order calculus has entered a brand new developing stage and has been already successfully applied in many fields of humanism social science researches except physics and engineering [6-9]. Fractional calculus can often make up for the deficiency of integer order calculus. The ever-broadening applications of fractional calculus make us believe that the research progress would be greatly promoted as fractional calculus is introduced into this subject. So in this paper, we try to set up a fractional supervision game model to study the dynamical system of multiple stakeholders.

The rest of the paper is organized as follows. In Section 2, we start with some concepts and assumptions and formulate an ordinary supervision game model of multiple stakeholders, which are necessary for future discussions. Section 3 contains some preliminaries of fractional calculus theory and introduces the Adams-Bashforth-Moulton (ABM) predictorcorrector approach, which are used in the next section. In Section 4, we set up a fractional game model to characterize the gambling process of those multiple stakeholders by the fractional order calculus; then we show an example and carry out numerical simulations. Phase diagram and time series graph are used to numerically simulate and analyse the dynamical process of the gambling system. We finally conclude our paper in Section 5. 


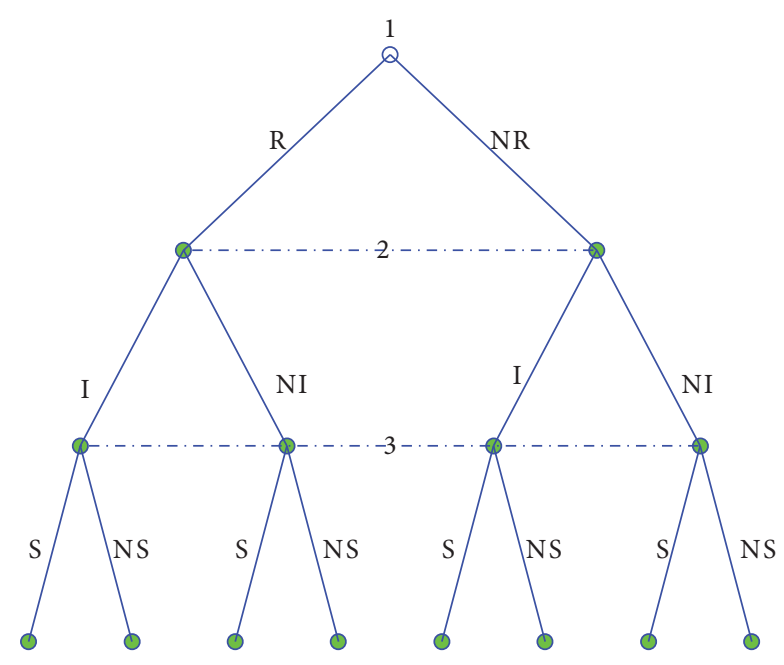

FIGURE 1: Network planning of the supervision game.

\section{Assumption and Formulation of the Ordinary Supervision Game Model of Multiple Stakeholders}

As we have introduced in the first section, there are many practical examples that can be abstracted into our proposed regulatory problem model. In this paper, we take the case of supervision game in colliery safety management system for example. There are actually two reasons for it: one is for the convenience and pertinence of narration; the other is that safety management is always the difficult and important factor of colliery management in China. As great dangers lie in coal industry, it is very urgent and necessary to decrease accident rate and improve our level of security management.

The gambling system of colliery safety management involves three interest groups from different levels, that is, coal mining workers, inspection team of the coal enterprise, and supervision department of the government, labeled as player I, player II, and player III, respectively. In order to build the game model, some assumptions and notations are given as follows (see Figure 1).

(1) Player I (coal mining workers) has two antagonistic strategies, doing jobs in the right way (i.e., operation behaviors complying with referenced regulatory requirements, standards, and codes, denoted as "R") or vice versa (some operation behaviors are not proper, even wrong, denoted as "NR"). We assume that the probability of choosing the strategy " $\mathrm{R}$ " is $x$ and $1-x$ for choosing the strategy NR, where $x \in[0,1]$. The job cost or input is $a_{1}$ when strategy " $R$ " is chosen, or else the job cost is $a_{2}$. The parameters $a_{1}$ and $a_{2}$ (and some other similar parameters mentioned below) are percentages of 1 unit industrial output.

(2) Player II (inspection team) also has two antagonistic strategies, inspection and no inspection, denoted as "I" and "NI," respectively. The cost of inspection is $b$, and player I (mining workers) would be fined $k_{1}$ for choosing "NR." The probability of choosing the strategy "I" is $y \in[0,1]$.

(3) The game strategies of player III (supervision department of the government) are supervision or no supervision, denoted as "S" and "NS." If III found some mistakes of I but II takes no inspection, I and II should pay fines of $k_{1}$ and $k_{2}$, respectively. The cost of supervision is $c$, and the probability of choosing supervision strategy " $\mathrm{S}$ " is $z \in[0,1]$.

Considering the actual situation, we assumed that the parameters mentioned above satisfy $0<b \leq c<a_{2}<a_{1}<$ $1<k_{1} \leq k_{2}$.

Based on the decision tree shown in Figure 1, we obtain the expection payoff (EP) of the three players. The EP of player I is

$$
u=x\left(1-a_{1}\right)+(1-x)\left[1-a_{2}-y k_{1}-(1-y) z k_{1}\right] .
$$

The EP of player II is

$$
v=y\left[(1-x) k_{1}-b\right]-(1-x)(1-y) z k_{2} .
$$

The EP of player III is

$$
w=z\left[(1-x)(1-y)\left(k_{1}+k_{2}\right)-c\right]
$$

By the first-order optimality condition, we get

$$
\begin{aligned}
-a_{1}+a_{2}+y k_{1}+(1-y) z k_{1} & =0, \\
(1-x)\left(k_{1}+z k_{2}\right)-b & =0, \\
(1-x)(1-y)\left(k_{1}+k_{2}\right)-c & =0 .
\end{aligned}
$$

The mixed strategy Nash equilibrium (MSNE) is given by the solution of three equations above, which is the optimal game strategy choice as we assume that all the participants are fully rational $[2,10]$. The MSNE point is not described in detail in this paper because the formula is so complicated that it is hard to know the real meaning of the results, and we prefer to believe that people are bounded rational in the reality.

\section{Preliminaries}

3.1. Fractional Order Calculus. As irregularities are common in nature and fractal theory has been highly realized in many fields of nonlinear science in nowadays, fractional order calculus is increasingly used as a kind of mathematical tool to describe exactly the essence of the problem. There are several commonly used definitions of fractional order derivative $[9,11-16]$. We utilize Caputo fractional order derivative in this paper. In contrast to the Riemann-Liouville fractional derivative, when solving differential equations using Caputo's definition, it is not necessary to define the fractional order initial conditions. The fractional derivatives of a continuous function $u(t)$ are defined by

$$
{ }_{t_{0}} D_{t}^{\mu} u(t)=\frac{1}{\Gamma(n-\mu)} \frac{d^{n}}{d t^{n}} \int_{t_{0}}^{t}(t-\tau)^{n-\mu-1} u(\tau) d \tau,
$$

where $n$ is the smallest integer number, larger than $\mu$.

3.2. Predictor-Corrector Approach for Fractional Order Calculus. Several numerical methods can be used to solve the fractional differential equations [14, 17-24]. Among them, 
Adams-Bashforth-Moulton (ABM) predictor-corrector approach has the merits of high precision and good stability, which was developed by Diethelm and other researchers $[18,25,26]$ and successfully applied to many systems, such as $[12,14,19,27]$. We take a fractional differential equation with initial conditions:

$$
\begin{aligned}
t_{0} D_{t}^{\mu} u(t) & =f(t, u(t)) \\
u_{t_{0}}^{(i)} & =u_{0}^{(i)}, \quad i=1,2, \ldots, n-1 .
\end{aligned}
$$

System (6) is equivalent to following Volterra integral equation:

$$
u(t)=\sum_{i=0}^{n-1} u_{0}^{(i)} \frac{t^{i}}{i !}+\frac{1}{\Gamma(\mu)} \int_{t_{0}}^{t}(t-\tau)^{n-\mu-1} f(\tau, u(\tau)) d \tau
$$

Set the uniform grid $\left(t_{n}=n h, n=1,2,3, \ldots, N\right)$ for some integer $N, h=T / N$ and assume that there is a unique solution of the function $u(t)$ on some interval $[0, T]$; system (6) can be approximated to the following difference schemes:

$$
\begin{aligned}
& u\left(t_{n+1}\right)=\sum_{i=0}^{n-1} u_{0}^{(i)} \frac{t_{n+1}^{i}}{i !}+\frac{h^{\mu}}{\Gamma(\mu+2)}\left[u\left(t_{n+1}, u^{p}\left(t_{n+1}\right)\right)\right. \\
& \left.+\sum_{i=0}^{n} a_{j, n+1} f\left(t_{j}, u\left(t_{j}\right)\right)\right],
\end{aligned}
$$

where

$$
\begin{aligned}
& a_{j, n+1} \\
& = \begin{cases}n^{\mu+1}-(n-\mu)(n+1)^{\mu}, & j=0 \\
(n-j+2)^{\mu+1}+(n-j)^{\mu+1}-2(n-j+1)^{\mu+1}, & 1 \leq j \leq n \\
1, & j=n+1 .\end{cases}
\end{aligned}
$$

The Adams-Moulton (AM) corrector method is formed by Formulae ((8) and (9)). Then, the predicted value $u^{P}\left(t_{n+1}\right)$ is determined by the Adams-Bashforth $(\mathrm{AB})$ method:

$$
u^{p}\left(t_{n+1}\right)=\sum_{i=0}^{n-1} u_{0}^{(i)} \frac{t_{n+1}^{i}}{i !}+\frac{1}{\Gamma(\mu)} \sum_{i=0}^{n} b_{j, n+1} f\left(t_{j}, u\left(t_{j}\right)\right),
$$

where

$$
b_{j, n+1}=\frac{h^{\mu}}{\mu}\left[(n+1-j)^{\mu}-(n-j)^{\mu}\right] .
$$

So, the Adams-Bashforth-Moulton (ABM) predictorcorrector approach for fractional order calculus has been achieved by formulae (8), (9), (10), and (11). The error of this numerical algorithm is expected as $O\left(h^{\nu}\right)$, where

$$
\nu=\min \{2,1+\mu\} \text {. }
$$

The two parts of ABM predictor-corrector approach, that is, the predictor method and the corrector method, are independent of each other. Almost any combination of them as we required can become a modifications of the algorithm $[14,18]$.

\section{Fractional Order Game Model and Numerical Simulation}

By the traditional game theory, replicator dynamics is an important concept to describe the evolutionary advantage of population in evolutionary game theory [2, 10, 28-30]. The evolutionary advantage of a population is reflected in the proportion of participants; that is, the growth rate of each player ( $\dot{x} / x$ for player I) equals to the difference between its adaptability and average adaptability, while the adaptability is represented as ER in our study. The most general form of replication equation is

$$
\frac{\dot{x}_{i}}{x_{i}}=\left[f_{i}\left(x_{1}, x_{2}, \ldots, x_{n}\right)-\sum_{i=1}^{n} x_{i} f_{i}\left(x_{1}, x_{2}, \ldots, x_{n}\right)\right] \text {, }
$$

where, $\left(x_{1}, x_{2}, \ldots, x_{n}\right)$ is the vector of the distribution of types in the population, $\dot{x}_{i} / x_{i}=d \ln x_{i} / d t$ represents the growth rate of type $i$, and the right side of (13) is the difference between the fitness of type $i$ and the average population fitness, which reflects the evolutionary advantage of type $i$.

So in the general way, the following replication equations are obtained:

$$
\begin{aligned}
\frac{\dot{x}}{x} & =\left.u\right|_{x=1}-u \\
& =(1-x)\left[-a_{1}+a_{2}+y k_{1}+(1-y) z k_{1}\right], \\
\frac{\dot{y}}{y} & =\left.v\right|_{y=1}-v=(1-y)\left[(1-x)\left(k_{1}+z k_{2}\right)-b\right], \\
\frac{\dot{z}}{z} & =\left.w\right|_{z=1}-w \\
& =(1-z)\left[(1-x)(1-y)\left(k_{1}+k_{2}\right)-c\right] .
\end{aligned}
$$

4.1. The Fractional Order Game Model of Colliery Safety Management. While Caputo fractional order derivative is substituted for ordinary integer derivatives, a fractional order game model is set up as follows.

$$
\begin{aligned}
& { }_{a} D_{t}^{\mu_{1}} x=x(1-x)\left[-a_{1}+a_{2}+y k_{1}+(1-y) z k_{1}\right], \\
& { }_{a} D_{t}^{\mu_{2}} y=y(1-y)\left[(1-x)\left(k_{1}+z k_{2}\right)-b\right], \\
& { }_{a} D_{t}^{\mu_{3}} z=z(1-z)\left[(1-x)(1-y)\left(k_{1}+k_{2}\right)-c\right] .
\end{aligned}
$$

In this part, the ABM predictor-corrector approach will be used to solve the fractional dynamical system (15) with given initial values

$$
\left(x_{0}^{(i)}, y_{0}^{(i)}, z_{0}^{(i)}\right), \quad i=1,2, \ldots, n-1 .
$$

In order to make a more simple and intuitive qualitative analysis of the character of solutions, the phase diagram $[12,30,31]$ and time series graph $[12,32,33]$ will be used to depict and analyse the dynamic process of the fractional order game model. 


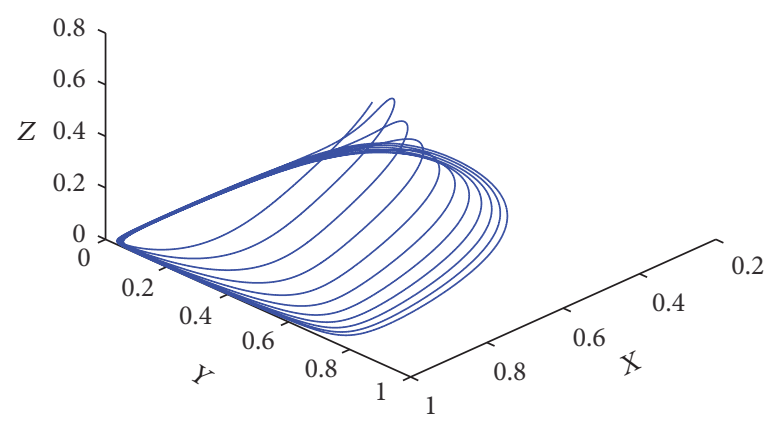

Figure 2: Phase diagram of fractional dynamical system $\left(\mu_{1}=\mu_{2}=\mu_{3}=0.95\right)$.

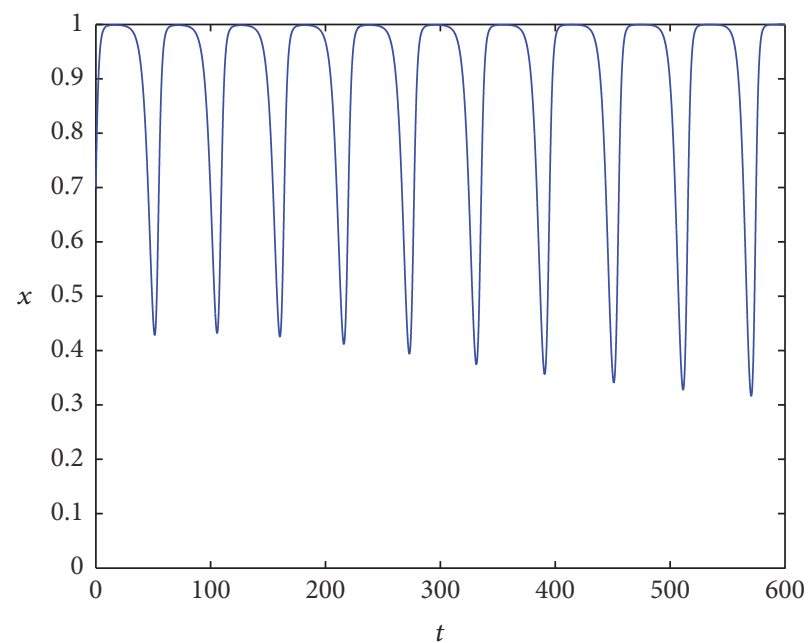

FIgURE 3: Time series graph of $x$ (Player I's proportion, $\mu_{1}=\mu_{2}=\mu_{3}=0.95$ ).

4.2. Numerical Simulation of the Fractional Game Model. According to the model assumptions made in Section 2, the parameters of the game model (15) and initial values of variables are supposed as

$$
\begin{aligned}
a_{1} & =1, \\
a_{2} & =0.2, \\
b & =0.2, \\
c & =0.25, \\
k_{1} & =k_{2}=2, \\
x_{0}^{(1)} & =0.7, \\
y_{0}^{(1)} & =0.5, \\
z_{0}^{(1)} & =0.6 .
\end{aligned}
$$

Formula (17) means if the labor cost of player I's normal work is 1 unit, his inactive or improper actions only cast 0.2 unit and the costs of player II's inspection and player III's supervision are 0.2 and 0.25 unit, respectively, and both player I and player II should pay fine of 2 units when their improper behaviors were found. Initial values of variables (see formula (18)) are the chances of their strategy selection at initial time.

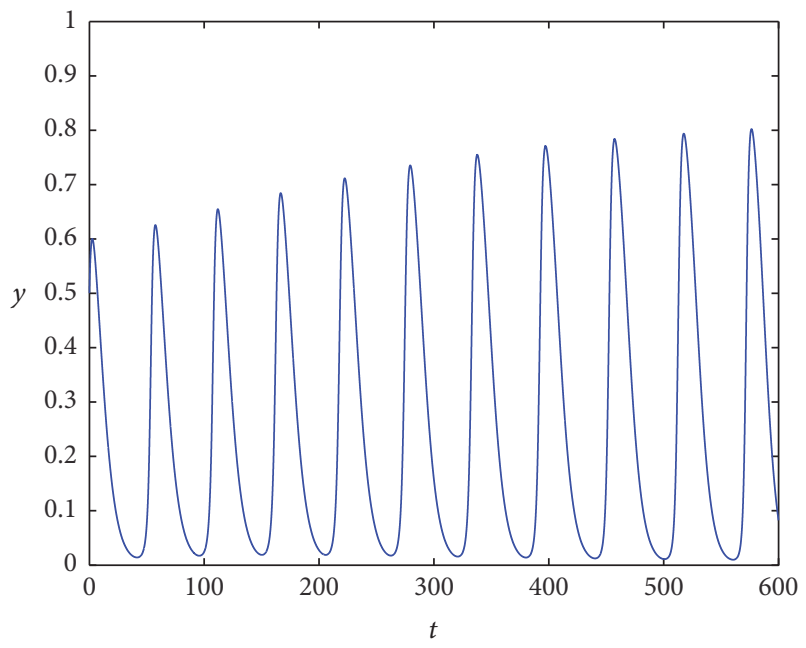

FIGURE 4: Time series graph of $y$ (Player II's proportion, $\mu_{1}=\mu_{2}=$ $\left.\mu_{3}=0.95\right)$.

Set $h=0.01, T=200 \mathrm{~s}, N=T / n, t_{0}=0$, and the fractional orders of differential equations are valued as $\mu_{k}=0.95, k=1,2,3$; the simulation results of the fractional game model are shown by the phase diagram (see Figure 2 ) and time series graphs (see Figures 3, 4, and 5). 


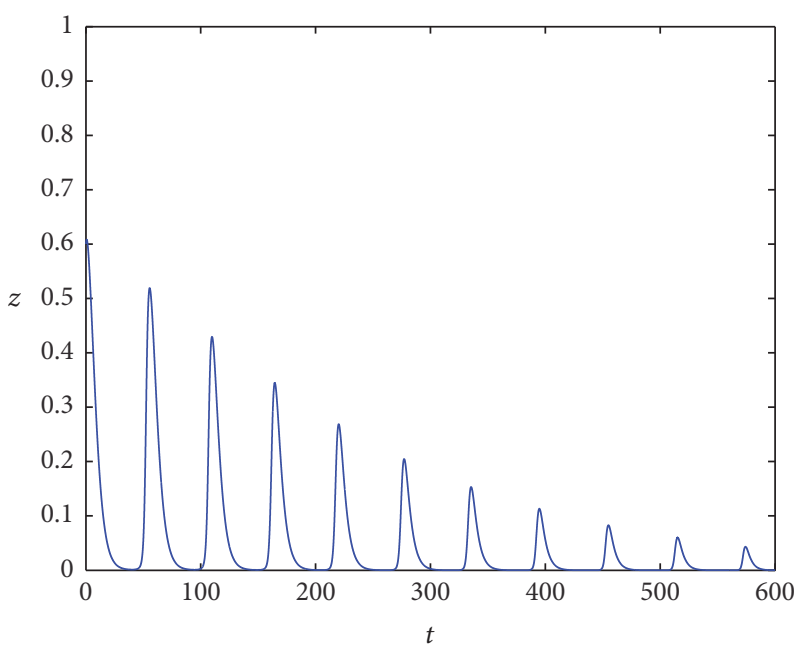

FIgURE 5: Time series graph of $z$ (Player III's proportion, $\mu_{1}=\mu_{2}=$ $\left.\mu_{3}=0.95\right)$.

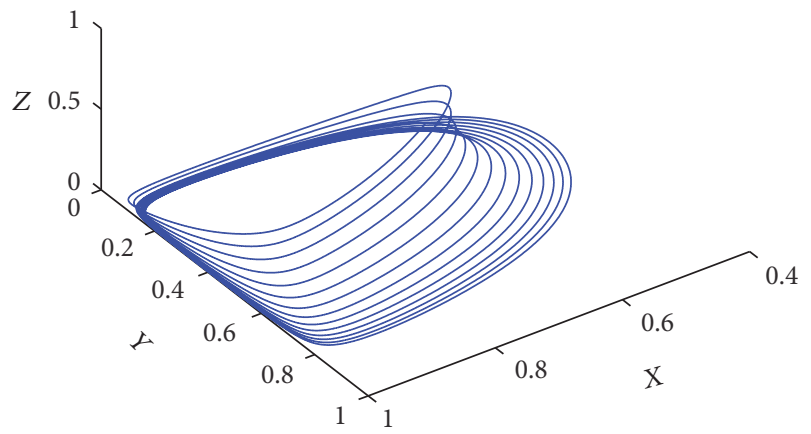

FIGURE 6: Phase diagram of fractional dynamical system $\left(\mu_{k}=\right.$ $1.05, k=1,2,3)$.

As the fractional orders are changed to $\mu_{k}=1.05, k=$ $1,2,3$, we obtain the similar phase diagram and time series graph (set $T=300$ for more distinct pictures), as shown in Figures 6, and 7.

Comparing the two groups of graphs (see Figures 2, 3, 4, 5, and Figures 6, 7), we could find out that the fractional order game model possesses similar dynamic features just as the one-order game model (see formula (14)). The trajectory of the dynamical game system is a closed curve around the center. The production safety input of workers, the intensity of the inspection, and the intensity of the government's supervision affect each other and form an interactive evolutionary process. In general, there is no evolutionary stable strategy (ESS) in this game model, and the dynamical system is not self-controlled. Therefore, it is necessary to restrain the behaviors of inspectors and supervisors.

Under certain conditions (see formulae (17), (18)), the proportion of player I choosing safe operation $(x)$ maintains at a certain height with high stability (see Figures 3 and 7), while the proportions of player II choosing safety inspection $(y)$ and of player III choosing the strategy of supervision $(z)$ remain a certain cyclical volatility (see Figures 4,5 , and 7 ). These results conform to the facts to a certain extent. It is noticeable that mining workers must operate safely at every

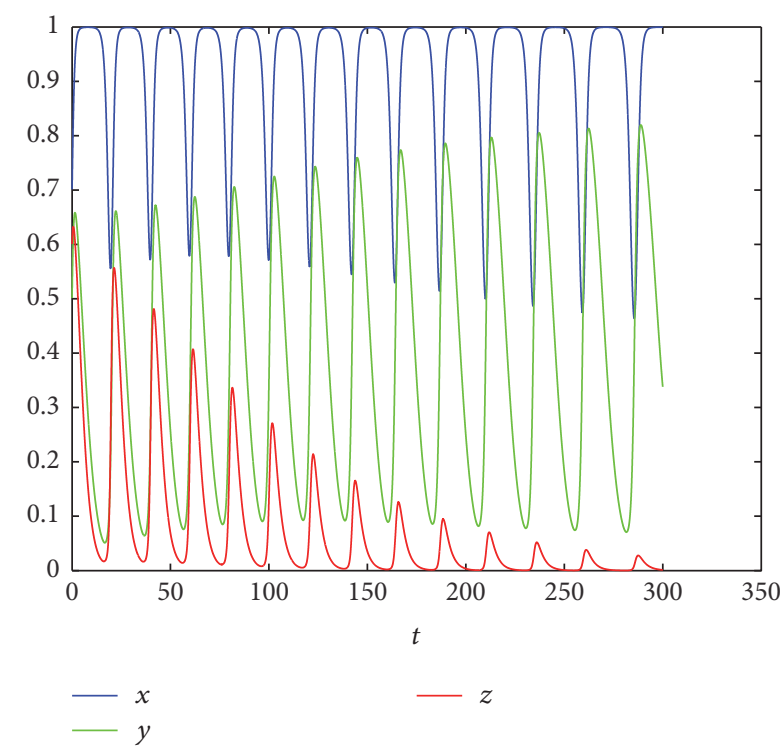

Figure 7: Time series graph of $x, y$, and $z\left(\mu_{k}=1.05, k=1,2,3\right)$.

moment. However, the inspections or supervision could be done periodically instead of being done all the time. It should be pointed out that the inspections or supervision must maintain a certain degree of level. Otherwise, the strategy of player I maybe changes sharply.

4.3. Some Special Cases of the Fractional Game System. Next, we consider three special cases; that is, there are rules and mandatory provisions on frequency of the inspections or the supervision, or both of them. Although these cases are special, they very likely occur in practice.

4.3.1. Fixing $y=y_{0}$. Firstly, let us fix $y=y_{0}$, which means there are mandatory provisions in the coal enterprises on the frequency of the inspections. Figure 8 shows the phase diagrams of choices of player I and player III ( $x$ and $z$ ) as we fix $y$ to $0.1,0.3$, and 0.4 , respectively. It can be concluded from their plots that along with the increase of $y$, the value $x$ will more likely approach to 1 . When $y$ exceeds a certain value, especially, $x$ will approach to 1 quickly and directly (see Figure 8(c)).

4.3.2. Fixing $z=z 0$. Secondly, let us fix $z=z_{0}$, which means there are rules on the frequency of supervision. Figure 9 shows the phase diagrams of choices of player I and player II $(x$ and $y)$ as we fix $z$ to $0.1,0.3$, and 0.5 , respectively. Some results of this case are very similar to the above case. It also can be concluded that $x$ will approach to 1 with the increase of $z$, and as $z$ exceeds some value, $x$ will approach to 1 quickly (see Figure 9(c)).

4.3.3. Fixing the Values of $y$ and $z$. Thirdly, let us fix $y=$ $y_{0}, z=z_{0}$, which means there are rules or mandatory provisions on both the frequency of inspections and supervision. Figure 10 shows some time series diagrams of $x$ (choices of player I) as we fix $z$ to 0.2 and fix $y$ to $0.2,0.25$, and 0.3 , 


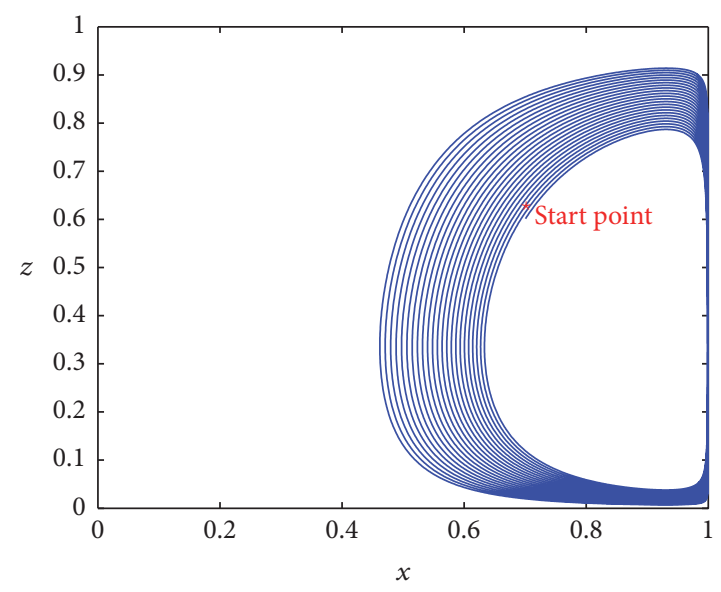

(a) Fixing $y=0.1$

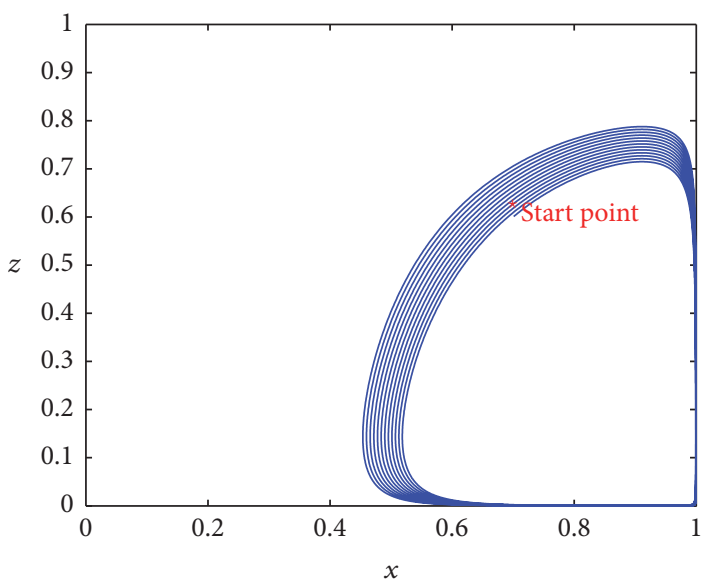

(b) Fixing $y=0.3$

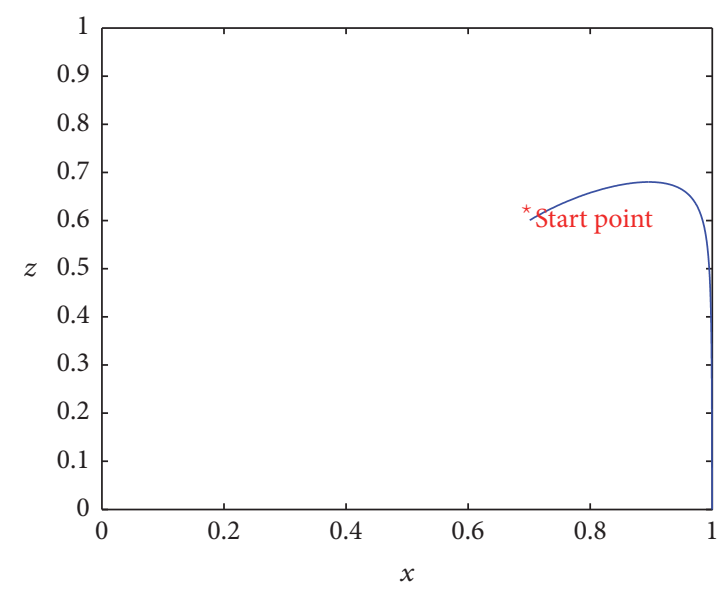

(c) Fixing $y=0.4$

FIGURE 8: Fixing $y=y 0$.

respectively. It can be concluded that $x$ will approach to 1 as long as $y$ and $z$ exceed some values.

By comparing the three graphics (see Figures 8, 9, and 10), we come to the conclusion that the effect of regulations and supervision would be more significant if there are rules or mandatory provisions on both the frequency of inspections and supervision.

\section{Conclusion}

In this paper, a fractional supervision game model of multiple stakeholders was built, which could be used to characterize a kind of typical problem in supervision management. The fractional model was solved by the ABM predictor-corrector calculation method. The methods of phase diagram and time 


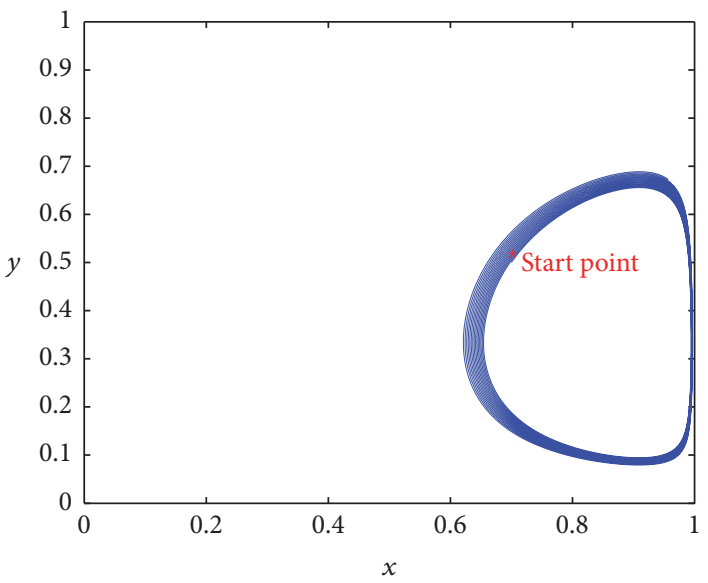

(a) Fixing $z=0.1$

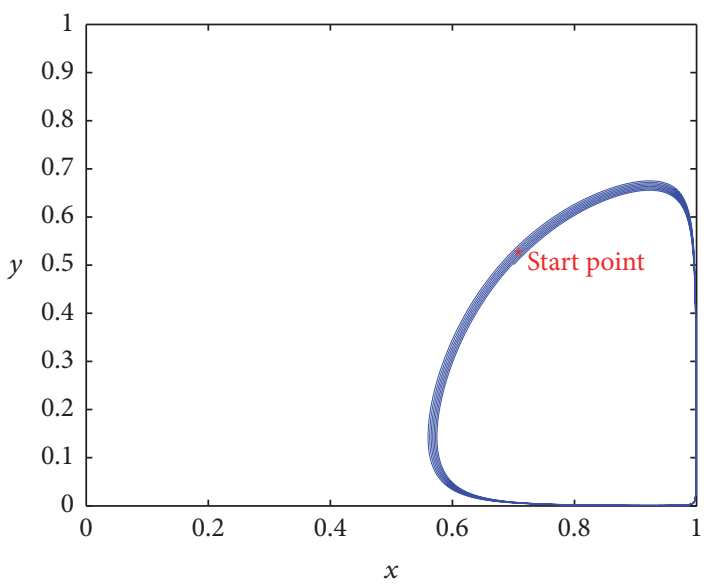

(b) Fixing $z=0.3$

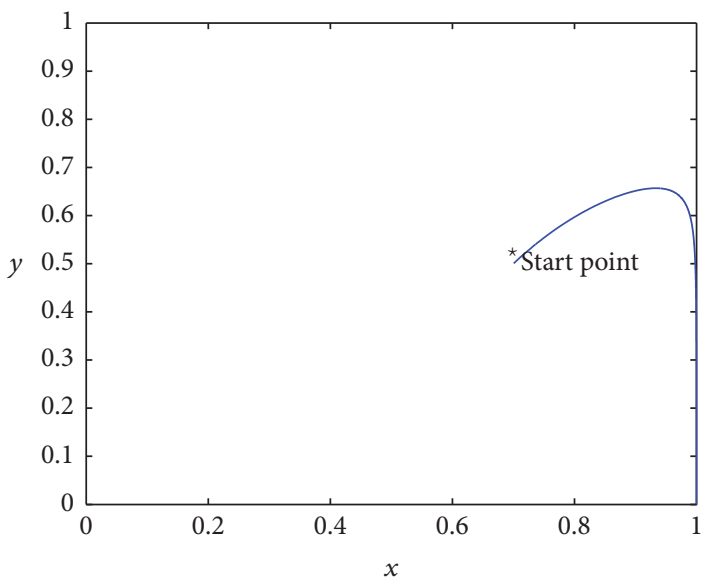

(c) Fixing $z=0.5$

FIGURE 9: Fixing $z=z 0$.

series graph were applied to simulate the dynamic process of the fractional order game model. Some results of numerical solutions analysed the behaviors of those interest groups related.

The results of the model show that the fractional order game model is scientific and reasonable and may provide new research methods and research directions for the original research of supervision management systems.

\section{Conflicts of Interest}

The authors declare that there are no conflicts of interest regarding the publication of this paper. 


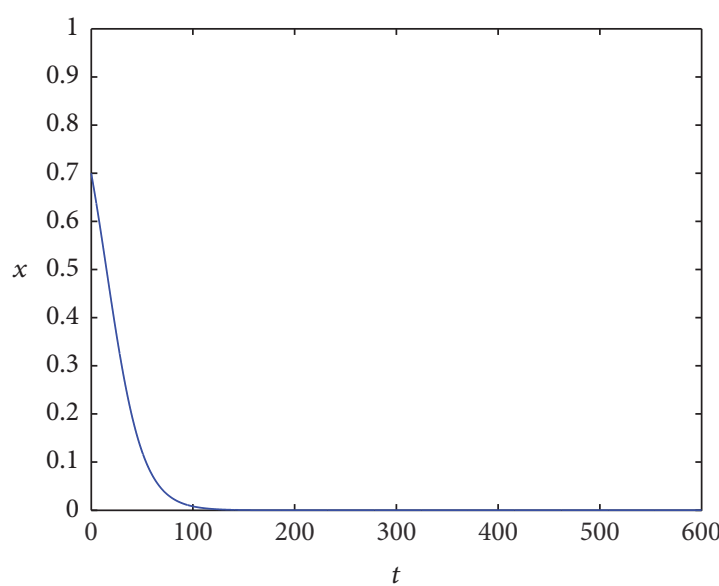

(a) Fixing $y=0.2, z=0.2$

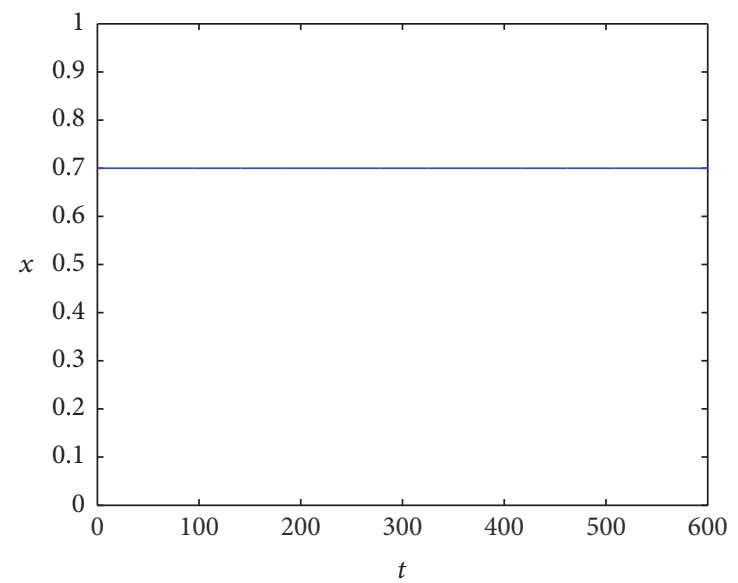

(b) Fixing $y=0.25, z=0.2$

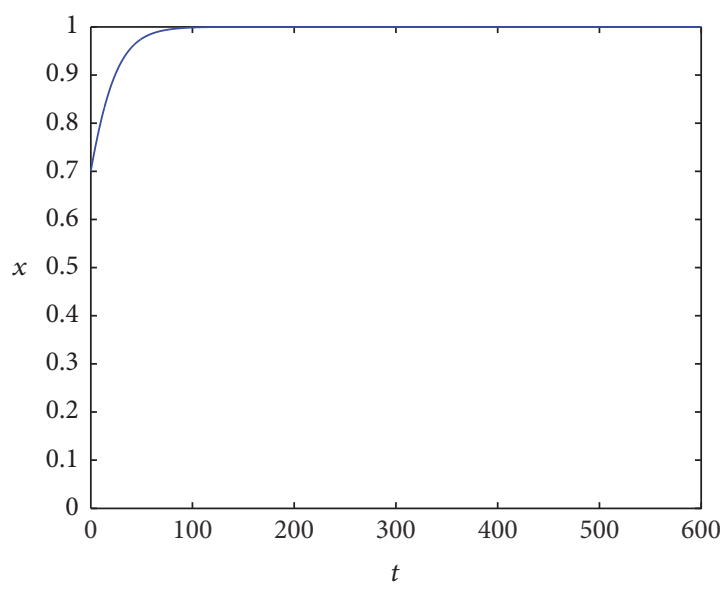

(c) Fixing $y=0.3, z=0.2$

FIGURE 10: Fixing $y=y_{0}$ and $z=z_{0}$.

\section{Acknowledgments}

This work is supported by National Nature Science Foundation of China (Grant no. 51574157): Research on Multilateral Game and Control Strategies in Coal Mine Safety System.

\section{References}

[1] J. Rasmussen, "Risk management in a dynamic society: a modelling problem," Safety Science, vol. 27, no. 2-3, pp. 183-213, 1997.

[2] R. Lu, X. Wang, and D. Li, "Extensive form game model of multiple interest groups in colliery safety," Journal of Qufu Normal University, vol. 39, no. 4, pp. 22-24, 2013.

[3] Y. Fernando, H. H. Ng, and Y. Yusoff, "Activities, motives and external factors influencing food safety management system adoption in Malaysia," Food Control, vol. 41, no. 1, pp. 69-75, 2014.

[4] R. Rocha, V. Mollo, and F. Daniellou, "Work debate spaces: a tool for developing a participatory safety management," Applied Ergonomics, vol. 46, pp. 107-114, 2015.

[5] O. Akintola and G. Chikoko, "Factors influencing motivation and job satisfaction among supervisors of community health workers in marginalized communities in South Africa," Human Resources for Health, vol. 14, no. 1, article 54, 2016.

[6] G. A. Anastassiou, Advances on Fractional Inequalities, SpringerBriefs in Mathematics, Springer, New York, NY, USA, 2011.

[7] J. A. Tenreiro Machado, "A probabilistic interpretation of the fractional-order differentiation," Fractional Calculus \& Applied Analysis, vol. 6, no. 1, pp. 73-80, 2003.

[8] A. A. A. Kilbas, H. M. Srivastava, and J. J. Trujillo, Theory and Applications of Fractional Differential Equations, vol. 204, North-Holland Publishing, 2006.

[9] I. Petráš, "Chaos in the fractional-order Volta’s system: modeling and simulation," Nonlinear Dynamics, vol. 57, no. 1-2, pp. 157-170, 2009.

[10] J. Maynard Smith, "The theory of games and the evolution of animal conflicts," Journal of Theoretical Biology, vol. 47, no. 1, pp. 209-221, 1974.

[11] Z. Bai, "On positive solutions of a nonlocal fractional boundary value problem," Nonlinear Analysis. Theory, Methods \& Applications, vol. 72, no. 2, pp. 916-924, 2010.

[12] B. Xin, J. Ma, T. Chen, and Y. Liu, "A fractional model of labyrinth chaos and numerical analysis," International Journal of Nonlinear Sciences and Numerical Simulation, vol. 34, no. 1, pp. 32-33, 2011. 
[13] Z. Bai and W. Sun, "Existence and multiplicity of positive solutions for singular fractional boundary value problems," Computers \& Mathematics with Applications, vol. 63, no. 9, pp. 1369-1381, 2012.

[14] L. Zhao and W. Deng, "Jacobian-predictor-corrector approach for fractional differential equations," Advances in Computational Mathematics, vol. 40, no. 1, pp. 137-165, 2014.

[15] H. H. Dong, B. Y. Guo, and B. S. Yin, "Generalized fractional supertrace identity for Hamiltonian structure of NLS-MKdV hierarchy with self-consistent sources," Analysis and Mathematical Physics, vol. 6, no. 2, pp. 199-209, 2016.

[16] A. Atangana and D. Baleanu, "New fractional derivatives with non-local and non-singular kernel: theory and application to heat transfer model," Thermal Science, vol. 20, no. 2, pp. 763769, 2016

[17] C. Yin, Y. Cheng, S.-M. Zhong, and Z. Bai, "Fractionalorder switching type control law design for adaptive sliding mode technique of 3D fractional-order nonlinear systems," Complexity, vol. 21, no. 6, pp. 363-373, 2016.

[18] K. Diethelm, N. J. Ford, and A. D. Freed, "A predictor-corrector approach for the numerical solution of fractional differential equations," Nonlinear Dynamics, vol. 29, no. 1-4, pp. 3-22, 2002.

[19] B. Yu and X. Jiang, "A fractional anomalous diffusion model and numerical simulation for sodium ion transport in the intestinal wall," Advances in Mathematical Physics, vol. 2013, Article ID 479634, 8 pages, 2013

[20] Z. Wang, "A numerical method for delayed fractional-order differential equations," Journal of Applied Mathematics, vol. 2013, Article ID 256071, 7 pages, 2013.

[21] J. Chen, Z. Zeng, and P. Jiang, "Global Mittag-Leffler stability and synchronization of memristor-based fractional-order neural networks," Neural Networks, vol. 51, pp. 1-8, 2014.

[22] Y. Cui, "Existence results for singular boundary value problem of nonlinear fractional differential equation," Abstract and Applied Analysis, vol. 2011, Article ID 605614, 9 pages, 2011.

[23] Y. Cui, "Uniqueness of solution for boundary value problems for fractional differential equations," Applied Mathematics Letters, vol. 51, pp. 48-54, 2016.

[24] Z. Bai, S. Zhang, S. Sun, and C. Yin, "Monotone iterative method for fractional differential equations," Electronic Journal of Differential Equations, vol. 2016, article 6, 2016.

[25] K. Diethelm, N. J. Ford, and A. D. Freed, "Detailed error analysis for a fractional Adams method," Numerical Algorithms, vol. 36, no. 1, pp. 31-52, 2004.

[26] K. Diethelm, "Increasing the efficiency of shooting methods for terminal value problems of fractional order," Journal of Computational Physics, vol. 293, pp. 135-141, 2015.

[27] B. Xin and Y. Li, "0-1 test for chaos in a fractional order financial system with investment incentive," Abstract and Applied Analysis, vol. 2013, Article ID 876298, 10 pages, 2013.

[28] J. W. Weibull, "Evolutionary game theory," Current Biology, vol. 9, no. 14, pp. 503-505, 1995.

[29] K. M. Page and M. A. Nowak, "Unifying evolutionary dynamics," Journal of Theoretical Biology, vol. 219, no. 1, pp. 93-98, 2002.

[30] R. Lu, X. Wang, and D. Li, "Application of multi-population evolutionary game in colliery safety and supervision," Mathematics in Practice and Theory, vol. 46, no. 19, pp. 31-36, 2016.

[31] R. Shone, Economic Dynamics: Phase Diagrams and Their Economic Application, Cambridge University Press, Cambridge, UK, 2nd edition, 2002.
[32] W. Zhao, T. Zhang, X. Meng, and Y. Yang, "Dynamical analysis of a pest management model with saturated growth rate and state dependent impulsive effects," Abstract and Applied Analysis, vol. 2013, Article ID 204642, 18 pages, 2013.

[33] X. Meng and L. Zhang, "Evolutionary dynamics in a LotkaVolterra competition model with impulsive periodic disturbance," Mathematical Methods in the Applied Sciences, vol. 39, no. 2, pp. 177-188, 2016. 


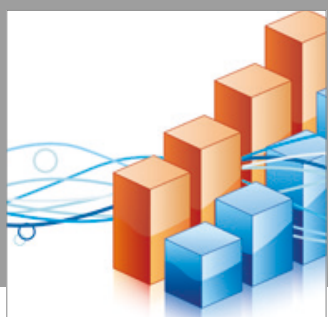

Advances in

Operations Research

vatersals

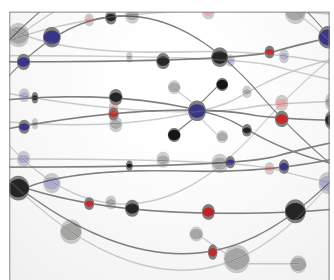

\section{The Scientific} World Journal
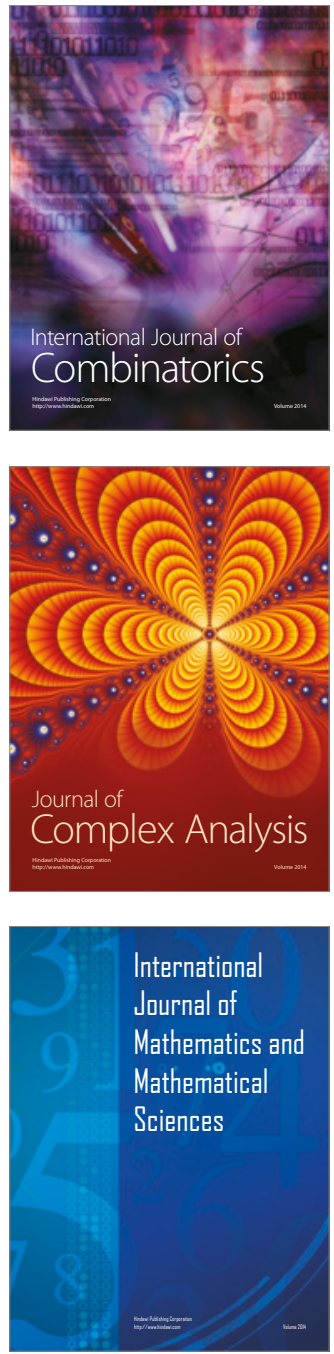
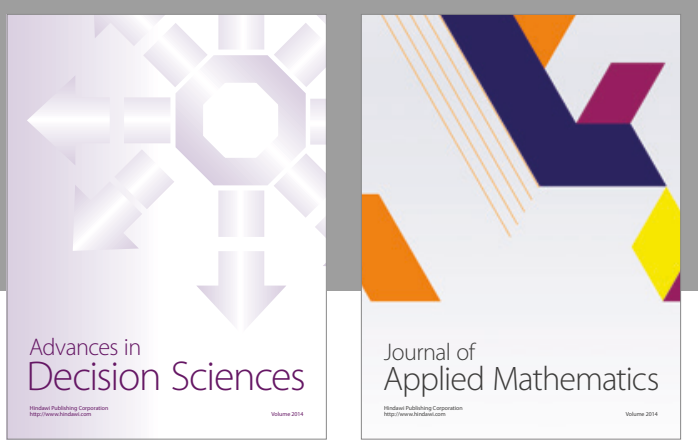

Algebra

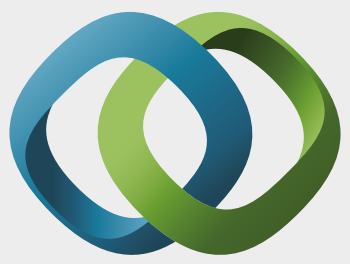

\section{Hindawi}

Submit your manuscripts at

https://www.hindawi.com
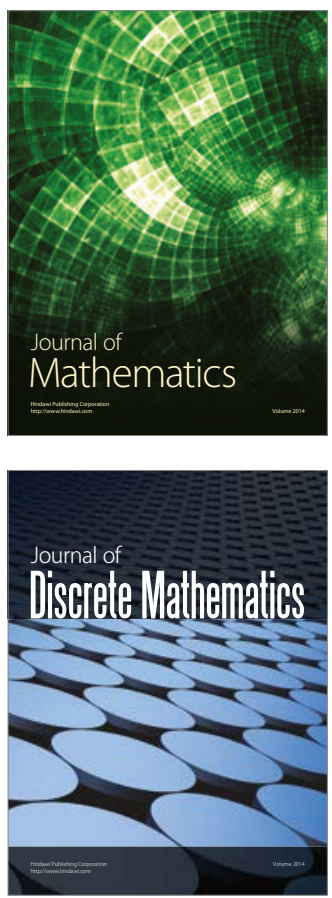

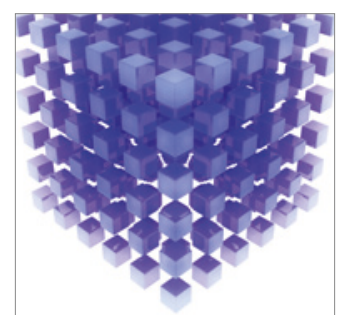

Mathematical Problems in Engineering
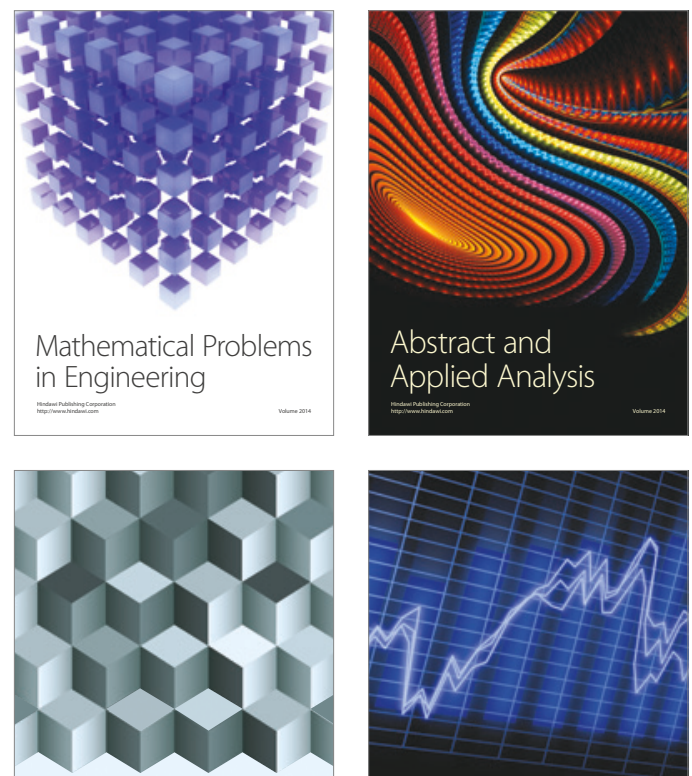

Journal of

Function Spaces

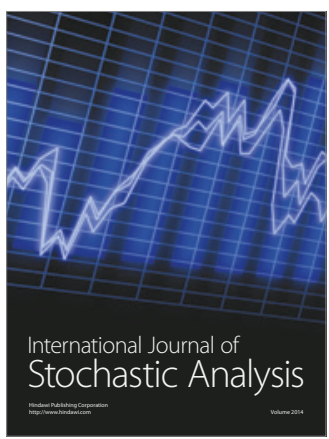

Probability and Statistics
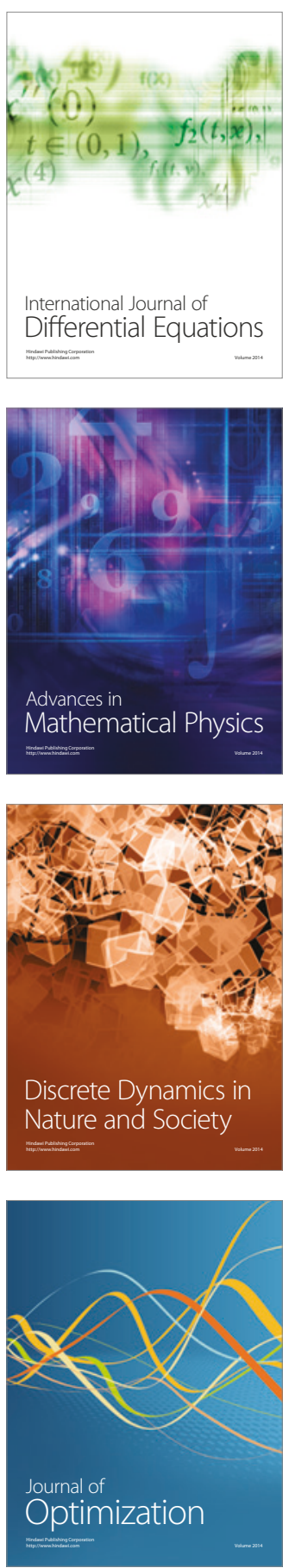